\title{
Long-term memory for concepts by squirrel monkeys
}

\author{
LEONARD E. BURDYN, JR., LINDA M. NOBLE, LORELEI E. SHREVES, \\ and ROGER K. THOMAS \\ University of Georgia, Athens, Georgia
}

\begin{abstract}
Memory for concepts learned previously in three separate experiments was assessed: (1) oddity and dimension-abstracted oddity after a 2.33-year interval, (2) conditional discrimination with conceptual simultaneous and successive cues after a 1.33-year interval, and (3) numerousness discrimination involving 2 versus 7 "dots" after a 5 -year interval. The strongest evidence for LTM was seen in the monkey retested on the 2 versus 7 dots problem on which he responded correctly on $80 \%$ of the first 40 trials. He also met criterion (36 of 40 correct) in 80 trials compared with 300 for his earlier training and with 240 and 360 trials for two monkeys being trained for the first time. There was also evidence that some other monkeys showed significant retention on some oddity and DAO tasks, but the evidence was less clear that a third set of monkeys had shown significant retention for the conditional discrimination task. Discussion considers the confounding of retention measures with the possibility for learning in LTM tasks as well as the need for more information on animals' LTM for concepts.
\end{abstract}

The squirrel monkeys in this laboratory have been subjects in a variety of studies involving conceptual behavior, reports on most of which were published between 1976 and 1983. Within the past year, some of these monkeys have been retested on some of those conceptual tasks. Reported here are the results of retesting (1) three monkeys approximately 2.33 years after training on a series of oddity and dimensionabstracted oddity tasks (see Thomas \& Frost, 1983), (2) four monkeys approximately 1.33 years after training on a conditional discrimination task in which both the simultaneous and the successive cues were exemplars of class concepts (see Burdyn, 1983), and (3) one monkey approximately 4.67 years after training on some numerousness judgment tasks (see Thomas \& Chase, 1980; Thomas, Fowlkes, \& Vickery, 1980).

Before considering the methods and results specific to these three types of tasks, it will be useful to address briefly what we mean by "concept" and "conceptual behavior." There is no standard definition of concept (see Heath, 1967; Kendler \& Kendler, 1975), but Thomas and his colleagues (e.g., Thomas, 1980; Thomas \& Kerr, 1976) have insisted that evidence for conceptual behavior in nonhuman animals must be such that it is impossible or highly unlikely that the animal's performance was based on the use of specific, as opposed to conceptual, cues. This distinction will be illustrated by the following example, which is relevant to the present study.

The order of authorship is alphabetical. Requests for reprints should be sent to Roger K. Thomas, Department of Psychology, University of Georgia, Athens, GA 30602.
The investigation of the oddity concept typically involves the presentation of three stimulus objects in a row, two of which are identical. The subject is reinforced for choosing the odd stimulus. Some investigators have presented the same three objects repeatedly (a task that Levinson, 1958, called the "oneodd" problem). In such cases, the subject might choose the odd stimulus on the basis of its specific physical properties, or it might have learned to respond to the object in terms of its position in the three typical, repeatedly presented, specific patterns (symbolically: AAB, ABA, BAA). It is possible that the subject responded on the basis of using the oddity concept, but that possibility is confounded by the specific property or pattern solutions.

In an attempt to eliminate the specific property solution by allowing each of the two types of objects to be alternately odd and nonodd, Robinson (1933) introduced what Levinson called the "twoodd" problem. However, she apparently overlooked the specific pattern solution (viz, in this case, AAB, ABA, BAA, BBA, BAB, ABB). Meyer and Harlow (1949) appear to have been the first to discuss these nonconceptual solutions, and they suggested that multiple problems be used and first-trial performances be emphasized when new problems were used. It should be noted that in the absence of firsttrial data analyses, it is possible to interpret improving performance over problems as evidence for the superiority in efficiency of the formation and use of learning sets to use of the oddity concept. Although learning set may reflect a kind of conceptual behavior, it does not necessarily provide evidence for the use of oddity per se. 
Thomas (1982) has also pointed to the confusing and conflicting terminology associated with the study of conceptual behavior, and has suggested (following Thomas \& Crosby, 1977) that concepts be defined in terms of the logical operations involved. Specifically, it was suggested that class concepts were those that involved only affirmation and its complement negation and that relational concepts also involved conjunctive, disjunctive, conditional, or biconditional operations and their respective complements. A distinction was also made between absolute and relative class concepts. With the former, the stimulus properties that may be used to affirm an exemplar as being a member of a class are inherent in the exemplar (e.g., the properties that identify a particular tree as a member of the class "tree"), but with the latter, the affirming cues are relative ones among the stimulus choices (e.g., "oddity" or "larger" or "fewer").

\section{LTM for Oddity and Dimension-Abstracted Oddity (DAO)}

There is a prior report of LTM for the "abstract concept" of "oddity" (Johnson \& Davis, 1973). However, two-odd problems without first-trial data analyses were used, and we question the validity of the study as evidence for LTM of the oddity concept. We do not question the impressive demonstration of LTM for learning set. After a 7-year interval, a group of 22-year-old rhesus monkeys (Macaca mulatta) had a mean of $80.7 \%$ correct during the first 7 days of LTM testing, and a group of 15-year-old monkeys had a mean of $70 \%$ correct. The difference between the two groups was consistent with the difference seen between them during original training.

The LTM data for conceptual oddity and DAO reported here were based on a series of tasks in which a new problem was given on each trial during original training (Thomas \& Frost, 1983) as well as during retention testing; hence, the nonceptual solutions are precluded. The three squirrel monkeys (Saimiri sciureus) had been trained originally on three oddity and three DAO tasks from September 1980 to April 1981. The LTM testing was done in August 1983approximately 2.33 years later. Logically considered, the six tasks increased in difficulty (except that the logical difficulty between Task 3 and Task 4 was unpredictable). We suggested the following nomenclature which may be used to analyze the nature of each task. In order of presumed easiest to most difficult (which was also the order of training), the tasks were OON3, O1N3, O2N3, OON2, O1N2, and OON1. The letters $O$ and $N$ are for odd and nonodd, respectively. The number following $O$ is the number of properties that the odd stimulus shared with the nonodd stimuli. The number following $\mathbf{N}$ is the number of properties shared by the nonodd stimuli and the odd stimulus when applicable. Thus, for example, O1N2 means that the nonodd stimuli in problems of this type shared two properties (color, form, size), and one of these was shared with the odd stimulus. Note that from trial to trial the particular types of properties shared were varied randomly. The positions of the objects were also varied randomly among the three locations. Objects were placed over foodwells, and the subject's displacement of the odd object revealed a reinforcer (currants). Finally, the distinction between oddity and DAO is that, with oddity, the nonodd objects are identical, but with DAO, they are not identical but share more properties with each other than they do with the odd object (the definition of DAO used here is based on, but is more general than, Bernstein's, 1961 , p. 243, original definition).

Following extensive pretraining on 10 one-odd problems (see Thomas \& Frost, 1983), the monkeys were trained to a $90 \%$ correct criterion ( 36 correct in a 40-trial session) on the conceptual OON3 task. They met criterion in a median of 1,160 trials. Subsequently, however, they were limited to 400 trials per task ( 10 sessions of 40 trials each) whether or not they reached criterion. One monkey met criterion on Task 2 (O1N3), and two monkeys met criterion on Task 4 (OON2). No monkey met criterion on Tasks 3 (O2N3), $5(\mathrm{O} 1 \mathrm{~N} 2)$, or 6 (OON1), although at least one monkey had several statistically significant "runs" of correct responses on Tasks 5 and 6. Table 1 gives the mean percentages of correct responses on the six oddity and DAO tasks used in the initial training.

For the test of LTM, each monkey was given 40 trials of each of the six types of tasks in random order at a rate of 40 trials per daily session. Following this, they were given 40 control trials. The control trials consisted, in random order, of 20 trials with three identical objects and 20 trials with three objects that differed in color, form, and size. These were insolvable problems, and it was expected that

Table 1

Mean Percentage Correct on Oddity and DAO Problems During Initial Training and During Testing for LTM Approximately 2.33 Years Later

\begin{tabular}{cccc}
$\begin{array}{c}\text { Type of } \\
\text { Problem }\end{array}$ & $\begin{array}{c}\text { Initial } \\
\text { Training }\end{array}$ & LTM & $\begin{array}{c}\text { Differed from } \\
\text { LTM Control }\end{array}$ \\
\hline & \multicolumn{3}{c}{ Oddity } \\
OON3 & 72.5 & 65 & Yes* \\
O1N3 & 78.3 & 59 & Yes** \\
O2N3 & 70.0 & 51 & No \\
& & DAO & \\
OON2 & 76.7 & 79.5 & Yes ${ }^{\dagger}$ \\
O1N2 & 65.8 & 62 & No \\
OON1 & 56.7 & 54 & \\
& Control for LTM & \\
& & 38.3 & \\
\hline
\end{tabular}

${ }^{*} p<.025 . \quad{ }^{* *} p<.05 . \quad t_{p}<.001$.


the monkeys would perform at chance levels (33.3\% correct), which they essentially did ( $38.3 \%$ correct).

The percentages correct for the LTM test on the six oddity and DAO tasks and on the control task are also given in Table 1. A one-way repeated measures analysis of variance showed a significant difference among the seven tasks $[F(6,12)=8.54, p<.001]$. Comparisons among tasks using the difference score method and following Keppel (1973, pp. 408-411) showed that performances on the OON3, O1N3, and OON2 tasks differed significantly from that on the control task (see Table 1). Additionally, comparisons between the LTM performances on OON3 and $\mathrm{O} 1 \mathrm{~N} 3$, between OON2 and O1N3, and between $\mathrm{O} 2 \mathrm{~N} 3$ and $\mathrm{O} 1 \mathrm{~N} 2$ differed significantly $(\mathrm{p}<.05)$.

The finding that the monkeys showed a higher mean percentage correct on Task 4 (OON2) than on Task $3(\mathrm{O} 2 \mathrm{~N} 3)$ during LTM testing was consistent with the results of their original training as was the lower mean percentage correct on Task 3 than on Task $2(\mathrm{O} 1 \mathrm{~N} 3)$. It appears that Task 4 is easier for squirrel monkeys to learn and remember than Task 3. This had not been anticipated because Task 3 was oddity and Task 4 was DAO. Interestingly enough, the performance of college students suggested that Task 4 was more difficult than Task 3 (Noble, 1983).

Retesting to assess LTM, as done here, confounds the purely memorial aspects of the performance with the possibility of relearning or new learning. Unfortunately, given the sequential nature of the original training versus the random presentation of the LTM-test problems, we do not have directly comparable data with which to assess savings on the LTM tests. We used random order in the LTM test to minimize the possibility of relearning, which might have occurred had we given the LTM problems in order of easiest to most difficult. It may be recalled that the monkeys had taken a median of 1,160 trials to learn the easiest of the six tasks originally. Thus, it is reasonable to suggest that the significant differences seen between performances on the LTM control task and three of the oddity/DAO tasks (with only 40 trials per task) provide significant savings and must have depended on memorial processses.

Finally, it may be noted that two of the three monkeys served in experiments during the interval between original and LTM training. One of them served as a subject in the experiment to be described in the next section. Given that it involved conceptual "sameness" and "difference," which some view as being akin to oddity, one might have suspected possible interference with LTM for oddity and DAO. Another monkey served in an experiment that reinforced conceptual judgments based on the number of sides of randomly constructed polygons (Terrell, 1983). There was no evidence in the performance of either monkey to suggest that this intervening training interfered with LTM for oddity and DAO.

\section{LTM for Conceptual Conditional \\ Discrimination}

The conditional discrimination paradigm involves one set of simultaneous discriminanda and one set of successive discriminanda. A member of the successive set is the cue to the correct choice among the items of the simultaneous set on a given trial (French, 1965). Riopelle and Copelan (1954) conducted an experiment in which rhesus monkeys responded correctly when the successive cues were conceptual. Thomas and Kerr (1976) reported an experiment in which squirrel monkeys responded correctly when the simultaneous cues were conceptual. Burdyn (1983) conducted an experiment in which squirrel monkeys responded correctly when both the simultaneous and the successive cues were conceptual. The LTM data to be reported here involved retesting the monkeys used by Burdyn.

The simultaneous discriminanda in Burdyn's study were a pair of identical objects, which were used as exemplars of the concept of "sameness," and a pair of objects that differed in color, form, or size and represented the concept of "difference." New objects or combinations of objects were used on each trial. The successive discriminanda in the last four of eight tasks were exemplars of the concepts of "triangularity" and "heptagonality."

An apparatus with three guillotine doors was used. A triangle or a heptagon was presented in the center door and exemplars of "sameness" and "difference" were presented, positioned randomly, behind the two side doors. A triangle was the cue that "sameness" was correct, and a heptagon was the cue that "difference" was correct.

Following pretraining (see Burdyn, 1983, for details), a series of eight tasks was given. Tasks 1-4 differed from Tasks 5-8 primarily in that the same triangle and heptagon were used throughout training on the first four tasks, but 120 triangles and 120 heptagons (randomly selected from trial to trial) were used in Tasks 5-8. In Task 1, the triangle was always presented, and responses to the "sameness" pair were always reinforced. In Task 2 , the heptagon was always presented, and responses to the "difference" pair were always reinforced. In Task 3, the triangle and heptagon were presented randomly (except that each appeared 15 times in a session) and the response to "sameness" or "difference" was reinforced depending on whether the triangle (which cued "sameness") or the heptagon ("difference") had been presented. Note that it was only on Task 3 that it became necessary to associate the successive cues with the simultaneous cues in order to receive reinforcement at criterion level. Task 4 differed from Task 3 only in that delays were used between withdrawal of the successive cue and presentation of the simultaneous cues. Initially, they were given a 0 delay, which meant that the successive cue was withdrawn con- 
currently with presentation of the simultaneous cues.

To this point in training, all monkeys met criterion on all tasks ( 27 correct in a 30-trial session on Tasks 1 and 2 and 13 correct of the 15 of the two subsets of problems in a 30-trial session on Tasks 3 and 4). The median trials to criterion was 360 on Task 1,375 on Task 2, 540 on Task 3 , and 330 on the 0 -delay condition of Task 4 . Following the 0 delay on Task 4 , the plan was to increase the delays exponentially $(1,2,4$, $8,16 \mathrm{sec}$, etc.) until a monkey failed to reach criterion in 300 trials on a given delay. The longest delay achieved by any monkey was $16 \mathrm{sec}$; the longest delays achieved by the other monkeys were 8,4 , and $2 \mathrm{sec}$.

Tasks 5-8 corresponded to Tasks $1-4$, except that exemplars of the concepts "triangularity" and "heptagonality" were used as the successive cues instead of the single triangle and single heptagon used in Tasks 1-4. The monkeys met criterion in medians of 330 trials on Task 5, 300 trials on Task 6, 300 trials on Task 7, and 225 trials on the 0 -delay condition of Task 8. On subsequent delays, the longest delay achieved by a monkey on Task 8 was $16 \mathrm{sec}$; the other three monkeys achieved delays of 8,4 , and $1 \mathrm{sec}$. The initial training of these monkeys on these tasks was completed June 9-15, 1982.

LTM testing of these monkeys was done from October 4 to October 11,1983 ; that is, approximately 1.33 years after completion of their initial training. The LTM test consisted of giving each monkey two 30-trial sessions of the 0-delay condition of Task 8 and two sessions with the longest delay that the monkey had achieved during his initial training on Task 8. In addition, two sessions of insolvable control trials were given in which the simultaneous cues were both exemplars of "sameness" or were both exemplars of "difference" and reinforcement was given randomly by position. Chance was $50 \%$, and the monkeys responded correctly on $40 \%$ of the control trials. The mean percentage of correct responses during LTM testing in the 0-delay condition was $66 \%$; in the previous-longest-delay condition, it was $58 \%$.

Using the number of correct responses, a one-way repeated measures analysis of variance showed a significant difference among the two delayed conditional discrimination tasks and the control task $[F(2,6)=6.93, p<.05]$. Comparisons among the means, using the difference score method and following Keppel (1973), showed a significant difference between the 0 -delay and control tasks $(p<.001)$, but the difference between the control task and their previous-longest-delay task was not significant. Based on these findings, a reasonable conclusion is that squirrel monkeys show significant LTM for a conceptual conditional discrimination task, but LTM for the task is impaired when a STM requirement is superimposed. Further research is needed both on
LTM for conceptual conditional judgments and on the interacting effects of STM and LTM on such judgments.

Finally, only one of the four monkeys had any training during the interval between the completion of training and the test of LTM for the conceptual conditional discrimination. That experience consisted only of shaping to respond in an automated testing apparatus (see next section). His data were between those of the "best" and the "worst" monkeys in terms of LTM for conceptual conditional discrimination. Thus, there was no evidence to suggest that the intervening experience had interfered with LTM.

\section{LTM for Conceptual Numerousness Judgments}

In this case, we are using a monkey (78-I-1) in a numerousness-numeral association study (in progress as this is written) that includes training similar to that which he had in two studies for which training was completed approximately 4.67 years ago (see Thomas et al., 1980; Thomas \& Chase, 1980). In the first of those studies he and another monkey were trained to choose the card with the fewer "dots." (Actually, the entities were black-filled circles of various diameters and at various locations on white index cards. The use of nonnumerousness cues such as specific patterns, cumulative area, or brightness differences was precluded by the large number of different stimuli used.) The cards were presented vertically in plastic card holders such that pushing against one caused it to swivel aside and reveal a currant reinforcer in a foodwell beneath. After pretraining, training was begun on a series of numerousness discrimination problems beginning with 2 versus 7 dots (hereafter the form 2:7 will be used to represent the discrimination problems). As a criterion of 45 correct in a 50trial session was reached on each problem, training proceeded in the order $2: 6,2: 5$, etc., to $3: 7,3: 6$, etc., until the problem 6:7 was reached. Upon reaching criterion on 6:7, which both monkeys did, training proceeded with $7: 8,8: 9$, and $9: 10$. On these last three problems, a maximum of 500 trials per problem was given. Both monkeys met criterion on $7: 8$, and $78-$ I-1, the monkey of interest for his LTM here, met criterion on 8:9. He did not make criterion on 9:10.

Following the completion of training on the Thomas et al. (1980) study, Monkey 78-I-1 was used in another study (Thomas \& Chase, 1980) in an attempt to determine whether squirrel monkeys could choose among three numerousness displays in terms of "fewest," "intermediate," and "most." The plan was to proceed in a stepwise manner towards the goal of having the monkeys make all three types of judgments concurrently, and a sequence of 13 tasks was planned. The training of Monkey 78-I-1 was terminated during the fifth task (see Thomas \& Chase for details), but two other monkeys performed much better (although neither completed the 13th task). 
Monkey 78-I-1 and two others (78-I-4 and 83-5) are now being trained in a study planned to investigate their ability to learn numerousness-numeral associations. At this time, they are being trained on numerousness discrimination problems comparable to those of Thomas et al. (1980). The principal differences are: (1) only a subset of the problems used by Thomas et al. is being used, and (2) the monkeys are now being trained in a fully automated apparatus, whereas the earlier study was done with a manually operated WGTA.

In the present study, which involves LTM for Monkey 78-I-1, the same stimuli are being used in the numerousness problems, except that they are presented as photographic slides. The stimuli are backprojected to a stimulus-response apparatus with nine separate panels (each $9 \mathrm{~cm}$ wide $\times 12 \mathrm{~cm}$ high) in three rows of three panels. At present, the top six and the bottom-center panels are covered and not being used. The stimuli are photographed such that one geometrical form (in the case of pretraining) or numerousness display appears on the lower left panel and another appears on the lower right panel. The position of the correct stimulus is determined randomly. To respond, the monkey presses on the panel. If the response is correct, the slide is terminated, a green light is illuminated just below the bottom-center panel, and reinforcement (a currant) appears in an aperture just below the green light. The currants are delivered via a carousel device which rotates to bring the currant within reach, when appropriate. An incorrect response causes the slide projector to back up to an empty slot (slides are loaded in every other slot) in order to readminister the same slide after the usual intertrial interval $(30 \mathrm{sec})$. Up to five such correction trials may be given, but only the response to the first presentation of a given slide is used in the data analysis. The operation of the equipment is controlled by a Commodore microcomputer. The data are also recorded and analyzed by the computer.

After shaping the monkeys to respond in the apparatus, they were given a pretraining discrimination problem involving a triangle and a circle. Upon reaching criterion (same as below) on this (which they did in a median of 120 trials), they were given the $2: 7$ discrimination problem. This was to be followed by 3:7, 4:7, 5:7, 6:7, and possibly $7: 8$ before training on numerousness-numeral associations was begun. To proceed from one problem to the next, the monkey would need to make criterion ( 36 correct in a 40 -trial session) and perform significantly better than chance $(p<.01)$ on a generalization test involving completely new stimuli (training was done with 80 slides per problem before the 40 new slides were presented in the generalization test).

The most compelling evidence for Monkey 78-I-1's LTM for the $2: 7$ task was that he made $80 \%$ correct responses in his first session of 2:7 after more than 5 years since he had last learned the $2: 7$ problem (the 4.67 years mentioned earlier was the time since the completion of his training for Thomas \& Chase, 1980). It may be recalled that he had had extensive training in which the fewer number of dots was correct. His LTM may reflect this relative judgment as opposed to his recall of 2:7 per se. Other evidence for his LTM may be seen in Table 2, which shows his trials to criterion on the $2: 7,3: 7$, and $4: 7$ problems 5 years ago as well as during retraining in 1984. Also shown, for comparison, are the data of Monkeys 78I-4 and 83-5, which were trained for their first time concurrently with 78-I-1's retraining.

Table 2 gives strong evidence for 78-1-1's LTM for the 2:7 task, when his 1984 performance is compared with that of his initial training as well as the performance of the other two monkeys. Table 2 also suggests that any advantage due to LTM that Monkey 78-I-1 might have had initially during retraining was overcome by the second task (3:7). Regarding the improvement of 78-I-4's and 83-5's performance on the 3:7 task, as compared with their performance on the 2:7 task, it may be noted that two types of learning may have transferred from the $2: 7$ to the 3:7 task. They may have learned to respond to displays with fewer dots or to avoid displays with seven dots, or both. The apparent decline shown by Monkey 83-5 on Task 4:7 is somewhat misleading. Although he took nine sessions to attain criterion on $4: 7$, he had $82.5 \%$ correct in the first session and he responded $80 \%$ correct or better in six of the sessions. In short, he was at a high level of correct responding, but not quite high enough to reach criterion until the ninth session. Similarly, $78-\mathrm{I}-1$ was above $80 \%$ correct on all three sessions of 4:7 in his 1984 retraining.

\section{General Discussion}

We are aware of only one other study (in addition to Johnson \& Davis, 1973, discussed earlier) involving LTM for concepts with a retention interval of greater than 1 year. Patterson and Tzeng (1979) readministered discrimination reversal problems to four lowland gorillas (Gorilla gorilla gorilla) 2.5 years after their initial training. The task was such that the authors concluded that the performances re-

Table 2

Trials to Criterion on Numerousness Discrimination Problems

for Monkey 78-I-1 from Thomas et al. (1980) and for Monkeys 78-1-1, 78-I-4, and 83-5 from a Study in Progress (1984)

\begin{tabular}{ccccc}
\hline & \multicolumn{4}{c}{ Monkeys/(Time of Training) } \\
\cline { 2 - 5 } Problems & $78-\mathrm{I}-1$ & $78-\mathrm{I}-1$ & $78-\mathrm{I}-4$ & $85-3$ \\
$(1980)^{*}$ & $(1984)$ & $(1984)$ & $(1984)$ \\
\hline $2: 7$ & 300 & 80 & 360 & 240 \\
$3: 7$ & 50 & 40 & 80 & 40 \\
$4: 7$ & 250 & 120 & 40 & 360 \\
\hline
\end{tabular}

*Data of publication; initial training completed May 22, 1979. 
flected "abstract concept" learning as opposed to "simple S-R learning." However, it is unclear how extensive the readministration of the problems was and to what extent relearning might have been confounded with retention.

The possible confounding of relearning with retention will be a factor in any study of retention. The act of readministering the same problems introduces the possibility, no matter how unlikely, that the animal simply was a rapid (re)learner. The more extensive the readministration of problems, the more likely that relearning will be a factor. To date, the clearest evidence for LTM of a concept appears to be provided by the performance of Monkey 78-I-1 on the 2:7 numerousness discrimination problem. That he was correct on $80 \%$ of the trials in his first session of the 2:7 problem suggests that relearning was not a significant factor. Even so, the possibility cannot be eliminated because he may have performed correctly by chance in the early trials (he was correct on the first four trials) and learned quickly what the contingencies were. On the other hand, he had not learned as quickly 5 years before, nor did his "controls" (78-I-4 and 83-5) learn quickly (see Table 2).

Similarly, the evidence seems compelling to us that the monkeys in the oddity-DAO study showed evidence of retention, but again, some degree of relearning was a possibility. Because of the lower-thanchance rate of performance on the control trials, we are less confident of the evidence for LTM in the conceptual conditional discrimination study; the lower-than-chance rate there was due largely to the fact that some extinction of responding was taking place.

Studies of LTM for concepts involving retention intervals of 1 year or more are likely to continue to be reported infrequently. Investigators who have the opportunity of obtaining such data should be encouraged to do so. It is our belief that many, perhaps most, mammals and, certainly, primates conduct their lives largely on the basis of responding to their environments conceptually. Much more information is needed regarding the capacities of nonhuman animals for concept use, acquisition, and retention.

\section{REFERENCES}

Bernstein, I. S. (1961). The utilization of visual cues in dimensionabstracted oddity by primates. Journal of Comparative and Physiological Psychology, 54, 243-247.
Burdyn, L. E., JR. (1983). Conditional discrimination with conceptual simultaneous and successive cues in the squirrel monkey. Unpublished master's thesis, University of Georgia, Athens.

French, G. M. (1965). Associative problems. In A. M. Schrier, H. F. Harlow, \& F. Stollnitz (Eds.), Behavior of nonhuman primates (Vol. 1, pp. 167-209). New York: Academic Press.

HeAth, P. L. (1967). Concept. In The encyclopedia of philosophy (Vol. 2, pp. 177-180). New York: The Free Press.

Johnson, C. K., \& DAvis, R. T. (1973). Seven-year retention of oddity learning set in monkeys. Perceptual and Motor Skills, 37, 920-922.

Kendle R, H. H., \& Kendle R, T. S. (1975). From discrimination learning to cognitive development: A neobehavioristic odyssey. In W. K. Estes (Ed.), Handbook of learning and cognitive processes: Vol. I. Introduction to concepts and issues. New York: Wiley.

KE PPEL, G. (1973). Design and analysis: $A$ researcher's handbook. Englewood Cliffs, NJ: Prentice-Hall.

LeVinson, B. (1958). Oddity learning set and its relation to discrimination learning set. Unpublished doctoral dissertation, University of Wisconsin, Madison.

Meyer, D. R., \& Harlow, H. F. (1949). The development of transfer of response to patterning by monkeys. Journal of Comparative and Physiological Psychology, 42, 454-462.

NoBle, L. M. (1983). Oddity and dimension-abstracted oddity $(D A O)$ in humans. Unpublished master's thesis, University of Georgia, Athens.

Pattenson, T. L., \& Tzeng, O. J. L. (1979). Long-term memory for abstract concepts in the lowland gorilla (Gorilla $\mathrm{g}$. Gorilla). Bulletin of the Psychonomic Society, 13, 279-282.

Riopelue, A. J., \& Copelan, E. L. (1954). Discrimination reversal to a sign. Journal of Experimental Psychology, 48, 143-145.

Robinson, E. W. (1933). A preliminary experiment on abstraction in a monkey. Journal of Comparative Psychology, 16, 231-236.

Terrell, D. F. (1983). Conceptual discrimination of polygons by squirrel monkeys. Unpublished master's thesis, University of Georgia, Athens.

ThомAs, R. K. (1980). Evolution of intelligence: An approach to its assessment. Brain, Behavior \& Evolution, 17, 454-472.

Thомаs, R. K. (1982). The assessment of primate intelligence. Journal of Human Evolution, 11, 247-255.

Thomas, R. K., \& Chase, L. (1980). Relative numerousness judgments by squirrel monkeys. Bulletin of the Psychonomic Society, 16, 79-82.

Thomas, R. K., \& Crosby, T. N. (1977). Absolute versus relative class conceptual behavior in squirrel monkeys (Saimiri sciureus). Animal Learning \& Behavior, 5, 265-271.

Thomas, R. K., Fowlkes, D., \& Vickery, J. D. (1980). Conceptual numerousness judgments by squirrel monkeys. American Journal of Psychology, 93, 247-257.

Thomas, R. K., \& Frost, T. (1983). Oddity and dimensionabstracted oddity (DAO) in squirrel monkeys. American Journal of Psychology, 96, 51-64.

Thomas, R. K., \& KERR, R. S. (1976). Conceptual conditional discrimination in Saimiri sciureus. Animal Learning \& Behavior, 4, 333-336.

(Manuscript received April 3, 1984; revision accepted for publication May 28,1984 .) 